\title{
Facile template-free hydrothermal synthesis and microstrain measurement of $\mathrm{ZnO}$ nanorods
}

\author{
L MOTEVALIZADEH, Z HEIDARY and M EBRAHIMIZADEH ABRISHAMI ${ }^{\dagger} * *$ \\ Department of Physics, Mashhad Branch, Islamic Azad University, Mashhad, Iran \\ ${ }^{\dagger}$ Department of Physics, Ferdowsi University of Mashhad, Mashhad 91775-1436, Iran
}

MS received 26 October 2012; revised 9 January 2013

\begin{abstract}
ZnO nanorods were synthesized at low temperature by hydrothermally heating $0 \cdot 1 \mathrm{M}$ solution of $\mathrm{ZnCl}_{2}$ for 5, 10 and $15 \mathrm{~h}$ at a $\mathrm{pH}$ of 10 . No template, seeded substrate, catalyst and autoclave were employed for the synthesis of $\mathrm{ZnO}$ nanorods. The effect of heating durations on the morphology and crystal orientation of the structure were investigated by using scanning electron microscopy and $\mathrm{X}$-ray diffraction, respectively. SEM images showed that the flower-like structures were formed in $5 \mathrm{~h}$ hydrothermally-heated sample, whereas the hexagonal zinc oxide nanorods were perfectly fabricated with the increase in growth time. XRD patterns showed that the preferred orientation in nanorods could be controlled by hydrothermal treatment time. The crystallite size and microstrain were analysed by Williamson-Hall and Halder-Wagner methods. These results revealed the presence of defects in $\mathrm{ZnO}$ nanorods. However, by increasing the hydrothermal treatment time, both defects in lattice and crystallite size are decreased.
\end{abstract}

Keywords. ZnO nanorod; hydrothermal process; microstrain; X-ray analysis; Williamson-Hall; HalderWagner.

\section{Introduction}

Zinc oxide based semiconductors have been studied extensively because of attractive properties: transparency, wide bandgap, strong room temperature luminescence, high electron mobility, carrier-induced ferromagnetism, etc (Hingorani et al 1993; Zhao et al 1997; Sakohara et al 1998; Sharma et al 2003). In recent years, nanostructured materials have been increasingly considered for new application areas and developing existing applications due to the large surface to volume ratio and quantum confinement effects for applications relying on heterogeneous reactions. In the previous works, we have fabricated $\mathrm{ZnO}$ based varistor (Ebrahimizadeh Abrishami et al 2012) and Kerr cell (Ebrahimizadeh Abrishami and Hosseini 2012) from nanostructures. To develop these applications, successful preparation of a variety of $\mathrm{ZnO}$ micro/nanostructures for specific purpose is necessary. In this case, the one-dimensional (1-D) $\mathrm{ZnO}$ nanostructures, nanorods in particular, hold much promise in optoelectronics (Liu et al 2010), gas sensors (Panda and Jacob 2009), solar cells (Yuan et al 2011), transparent electrodes (Kim et al 2011), etc. Because, the operations of nanorods base devices entail the length, cross-section diameter and orientation of rods, controlling size and morphology of the nanostructures is a significant cha-

*Author for correspondence (ebrahimizadeh@ymail.com) llenge to manage the conditions of fabricating process. Numerous methods have been established for the successful growth of $\mathrm{ZnO}$ nanorods such as chemical vapour deposition (Park et al 2002), microwave irradiation (Brahma et al 2010), vapour-liquid-solid growth (Huang et al 2001), carbothermal evaporation (Yao et al 2002), electrodeposition (Kim et al 2006), template basis synthesis (Li et al 2000), thermal evaporation (Umar et al 2006) and electrochemical deposition (Ko et al 2012). Among these techniques, preparing $\mathrm{ZnO}$ nanorods via hydrothermal route provides an easy and convenient method with high ability in controlling the physics of rods. Hydrothermal processing has some major differences from other techniques such as preparing the powders directly from solution, ability to control the shape and size of structures, being highly reactive in sintering and not needed in calcination and milling process (Sōmiya and Roy 2000). Hydrothermal method includes various techniques of crystallizing substances from high temperature aqueous solution on nucleating sites at high vapour pressures. For typical hydrothermal research, the crystal growth is performed in an apparatus consisting of a steel vessel called autoclave. A possible disadvantage of this method is the high cost of the autoclaves. In addition, $\mathrm{ZnO}$ nanorods can be usually grown on templates or a variety of substrates by affixing pre-synthesized seeds for better controlling the nucleating sites (He 2005; Lipowski et al 2007; Baruah and Dutta 2009). The template based hydrothermal method always requires complicated 
Table 1. Some reports on the hydrothermal method and the effect of the starting materials, types of nucleating sites and $\mathrm{pH}$ of the solution on morphology of the samples.

\begin{tabular}{|c|c|c|c|c|c|}
\hline Reference & Precursors & Nucleating site & $\mathrm{pH}$ & $\begin{array}{l}\text { Hydrothermal } \\
\text { temperature }\left({ }^{\circ} \mathrm{C}\right)\end{array}$ & Morphology \\
\hline Ghosh et al (2008) & $\begin{array}{l}\mathrm{Zn}\left(\mathrm{NO}_{3}\right)_{2} \cdot 6 \mathrm{H}_{2} \mathrm{O} \\
\left(\mathrm{CH}_{2}\right)_{6} \mathrm{~N}_{4}(\mathrm{HMT})\end{array}$ & $\mathrm{ZnO}$ nanoparticles on glass & $x$ & 95 & Nanorod \\
\hline Zhu et al (2007) & $\begin{array}{l}\mathrm{Zn}\left(\mathrm{NO}_{3}\right)_{2} \cdot 6 \mathrm{H}_{2} \mathrm{O} \\
\mathrm{HMT}, \mathrm{Na}_{3} \mathrm{C}_{6} \mathrm{H}_{5} \mathrm{O}_{7}\end{array}$ & $\mathrm{Zn}(\mathrm{OH})_{2}$ in solution & 11 & 85 & $\begin{array}{c}\text { Hollow } \\
\text { microsphere }\end{array}$ \\
\hline Sambath et al (2012) & $\mathrm{Zn}\left(\mathrm{NO}_{3}\right)_{2} \cdot 6 \mathrm{H}_{2} \mathrm{O}, \mathrm{HMT}$ & $\mathrm{Zn}(\mathrm{OH})_{2}$ in solution & $\begin{array}{c}5 \text { and } 6 \\
8 \\
9 \text { and } 10\end{array}$ & 90 & $\begin{array}{c}\text { Nanorod } \\
\text { Nanoflake } \\
\text { Nanoflower }\end{array}$ \\
\hline Miao et al (2012) & $\mathrm{Zn}\left(\mathrm{NO}_{3}\right)_{2} \cdot 6 \mathrm{H}_{2} \mathrm{O}$ & $\begin{array}{l}\mathrm{ZnO} \text { nanofibres on } \\
\mathrm{Si} \text { substrate }\end{array}$ & 11 & 90 & Nanorod \\
\hline Aneesh et al (2007) & $\mathrm{Zn}\left(\mathrm{CH}_{3} \mathrm{COO}\right)_{2} \cdot 2 \mathrm{H}_{2} \mathrm{O}$ & $\mathrm{Zn}(\mathrm{OH})_{2}$ in solution & 8 and 11 & 150 & Nanoparticle \\
\hline
\end{tabular}

synthetic procedure. High costs, difficulties in removing the template, small amounts of product and timeconsuming synthesis process are the major disadvantages of using templates as well as seeded substrates. Therefore, further development of easy, low temperature and template-free synthesis method is quite necessary. Hydrothermal growth mechanism of $\mathrm{ZnO}$ nanostructures depends on the growth conditions such as reaction temperature (Chen et al 1999), reaction concentration and time (Chander and Raychaudhuri 2006) and $\mathrm{pH}$ of the solution (Sambath et al 2012) (table 1).

Besides, the crystal imperfections can affect the properties of nanostructures. An ideal crystal would extend infinitely in all the directions; therefore, no crystals are perfect due to their finite size. The effect of this deviation from perfect crystallinity changes the broadening of diffraction peaks. These broadenings are used to calculate the two main properties: crystallite size and lattice strain. Crystallite size is a measure of the size of coherently diffracting domains. Lattice strain is a measure of the distribution of lattice constants arising from crystal imperfections including the grain boundary triple junction, contact or sinter stress, stacking faults and coherency stress. Hydrothermal treatment time also affects the structure of crystal, the lattice parameters and the mechanical properties such as strain and stress. Both crystallite size and lattice strain have effects on Bragg peaks by increasing the peak width, intensity and shifting the $2 \theta$ peak position. Williamson-Hall $(\mathrm{W}-\mathrm{H})$ analysis simplified integral breadth methods where size- and straininduced broadening are deconvoluted by considering the peak width as a function of $2 \theta$. Although X-ray profile analysis is an averaging method, it still holds a dominant position in crystallite size determination. By using $\mathrm{W}-\mathrm{H}$ analysis, Navaladian et al (2009) showed that the defect density was higher in $\mathrm{Ag}$ nanosphere when compared to nanorods. In addition, Dhara and Giri (2011) observed that the crystal strain of $\mathrm{ZnO}$ nanorod prepared by a mechanochemical method decreased with the increase in milling time.

In this paper, we present a template-, catalyst- and autoclave-free hydrothermal method to synthesize $\mathrm{ZnO}$ nanorods. Comparing to the conventional hydrothermal method, the present synthesis process has the advantage of simplicity and low cost (without using any special equipments, templates or substrates), low growth temperature $\left(90^{\circ} \mathrm{C}\right)$, controlling morphology and crystallization orientation with hydrothermal treatment time duration, high morphological yield (nearly 100\%) and low crystal strain. In addition, a comparative study of the mean crystallite size of the compounds obtained from powder XRD is reported. The strain due to lattice deformation of $\mathrm{ZnO}$ nanorods was estimated by Halder-Wagner method and modified forms of $\mathrm{W}-\mathrm{H}$ analysis, viz. uniform deformation model (UDM), uniform stress deformation model (USDM) and uniform deformation energy-density model (UDEDM). UDEDM model provides information on the stress-strain relation and strain as a function of energy density to show the importance of strain contribution in XRD broadening.

\section{Experimental}

Zinc oxide nanorods were synthesized successfully using zinc chloride, $\mathrm{ZnCl}_{2}$ (Aldrich, 98\%). 0.1 M zinc chloride was dissolved in distilled water and stirred for $30 \mathrm{~min}$. The initial solution of deionized water and zinc chloride was stirred for half an hour. Ammonia $\left(\mathrm{NH}_{3}\right)$ was added to the solution dropwise to increase the $\mathrm{pH}$ to 10 . Then, the mixture was transferred to a three-necked flask instead of the autoclave system. The three-necked flask was equipped with an Ar gas inlet tube, a condenser and a thermometer. The mixture was hydrothermally heated at 
$90{ }^{\circ} \mathrm{C}$ heat bath in the presence of Ar gas. The precipitate was observed when the temperature increased to $90{ }^{\circ} \mathrm{C}$ but for the complete precipitation, the mixture was hydrothermally heated at $90^{\circ} \mathrm{C}$ for different times $(5,10$ and $15 \mathrm{~h})$. Thereafter, the white precipitate was washed with distilled water and dried at $40^{\circ} \mathrm{C}$.

In this work, the crystal structure of the synthesized powders was characterized by using X-ray diffraction (XRD) technique. The crystallite size and lattice strain were estimated from broadening of XRD peaks by using Scherrer, modified Williamson-Hall and Halder-Wagner methods. Scanning electron microscopy (SEM) micrographs were used to monitor the morphology of structures.

\section{Results and discussion}

\subsection{XRD analysis}

Figure 1 shows XRD patterns of the samples with different hydrothermal treatments for 5,10 and $15 \mathrm{~h}$. All the patterns were indexed as the hexagonal wurtzite structure and no other peaks were detected. The intense peaks in the patterns showed high crystallinity of the products. As can be seen in figure 1 , the nanorods are not aligned in certain direction and a random growth pattern is observed. The relative intensities of the reflection peaks in figures 1(a) and (c) agree well with XRD pattern of bulk $\mathrm{ZnO}$. On the other hand, with increasing hydrothermal treatment from 5 to $10 \mathrm{~h}$ and 10 to $15 \mathrm{~h}$, the preferred orientation of nanorods was changed from [101] to [002] and vice versa, respectively. Using the substrate (Kim et al 2006; Zhu et al 2008) and seeding the substrate (Baruah and Dutta 2009; Miao et al 2012) are generally effective methods to control the orientation of nanostructures. In this work, it is desired to establish the extent to which the hydrothermal treatment time plays a significant role in determining the orientation of nanorods. Figure 1(b) shows that preferable $c$-axis oriented $\mathrm{ZnO}$ nanorods can be grown by the substrate-free hydrothermal process, in spite of the other reports on synthesizing the same nanorods on the various substrates (Yuan et al 2011; Sima et al 2012). Therefore, the preferential growth of $\mathrm{ZnO}$ nanorod along [002] was controlled by the hydrothermal treatment time. In this report, it is suggested that there can be a critical hydrothermal treatment time for which the preferred orientation in [002] direction is maximum. We have obtained $i_{[002]}$ which is described as the ratio of [002] peak intensity to the sum of three main peaks intensities ([100], [002] and [101]) (Ebrahimizadeh Abrishami and Kompany 2012). The ratio $i_{[002]}$ plotted in figure 2 is a clear analysis of the orientation alignment of nanorods. It can be seen that the suggestive Gaussian fit on $c$-axis crystallization of $\mathrm{ZnO}$ nanorods data is maximized in the hydrothermal treatment time of about $11 \mathrm{~h}$.

\subsection{Crystallite size and mechanical properties}

3.2a Scherrer method: The broadening of XRD pattern is attributed to the crystallite size-induced or straininduced broadening. XRD profile analysis is a simple and powerful method to evaluate the crystallite size and lattice strain. Two factors determine the breadth of Bragg peak including instrument- and sample-dependant effects. To do an accurate analysis for size and strain effects, the instrumental broadening must be accounted for. To decouple these contributions, it is necessary to collect a diffraction pattern from the line broadening of a standard material such as silicon to determine the instrumental broadening. The instrument-corrected broadening $\beta_{\mathrm{D}}$ corresponding to each diffraction peaks was estimated by the relation $\beta_{\mathrm{D}}=\left(\beta_{\mathrm{obs}}^{2}-\beta_{\mathrm{inst}}^{2}\right)^{1 / 2}($ Rogers and Daniels 2002).

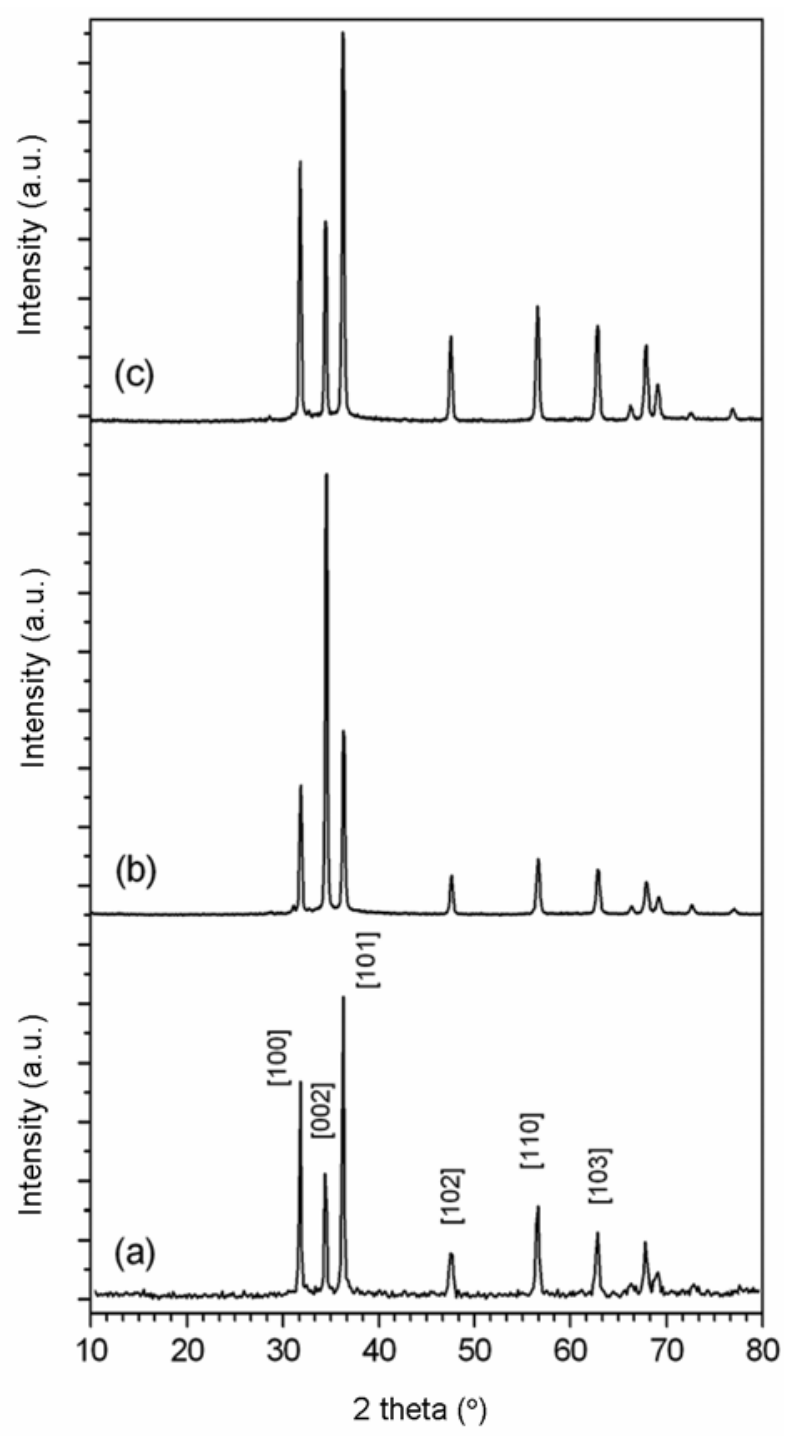

Figure 1. X-ray diffraction patterns of $\mathrm{ZnO}$ nanorods prepared after different hydrothermal heating times: (a) $5 \mathrm{~h}$, (b) $10 \mathrm{~h}$ and (c) $15 \mathrm{~h}$. 
The crystallite size of $\mathrm{ZnO}$ nanorods were determined from X-ray line broadening method by Scherrer's equation

$$
D=\frac{k \lambda}{\beta_{\mathrm{D}} \cos \theta} \Rightarrow \cos \theta=\frac{k \lambda}{D}\left(\frac{1}{\beta_{\mathrm{D}}}\right),
$$

where $D$ is the crystallite size in nanometers, $k$ the shape factor (0.94), the wavelength of $\mathrm{CuK} \alpha$ radiation and $\beta_{\mathrm{D}}$ the peak width at half maximum intensity. A plot is drawn with $1 / \beta_{\mathrm{D}}$ along the $x$-axis and $\cos \theta$ along the $y$ axis for $\mathrm{ZnO}$ nanorods as shown in figure 3 . In order to calculate the average crystallite size, three approaches including averaging directly through crystallite sizes obtained for three main peaks, estimating from the slope of the linear fit (Khorsand Zak et al 2011, 2012) and from the linear fit to the data through zero were considered (figure 3). The results obtained from the Scherrer method are summarized in table 2 . It can be clearly seen that there is an extreme deviance in the value of crystallite size estimated from the slope of the linear fit compared to the other two approaches. Furthermore, $y$-intercept in this fit has no physical meaning. Consequently, it seems that the average crystallite size estimated from the slope of linear fit through zero is coincident with that obtained by averaging directly and the results calculated from the slope of linear fit are inaccurate.

3.2b Williamson-Hall method: XRD peaks are broadened by small crystallite size and lattice distortion caused by lattice dislocations. However, effect of strain and imperfection on the line broadening differs from the

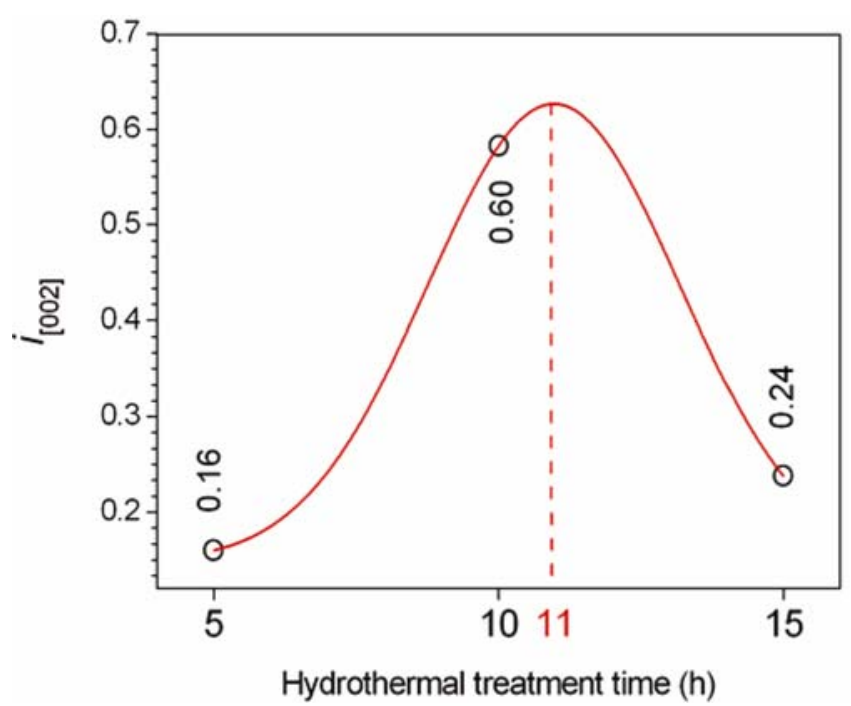

Figure 2. Ratio of [002] peak intensity to sum of three main peaks intensities ([100], [002] and [101]) plotted against hydrothermal treatment time. There is a suggestive critical time of hydrothermal heating when preferred growth along [002] direction is maximum. effect of crystalline size. The strain induced in $\mathrm{ZnO}$ nanorods was calculated using the relation (Stokes and Wilson 1944):

$$
\varepsilon_{\mathrm{str}}=\frac{\beta_{\mathrm{S}}}{4 \times \tan \theta}
$$

where $\varepsilon_{\text {str }}$ is the weighted average strain and $\beta_{\mathrm{S}}$ the integral breadth of reflection in radians located at $2 \theta$. Size and strain broadenings in (1) and (2) show a different $\theta$ that provides a way to separate each effect. It is possible to deconvolute each contribution by combining the two equations (Williamson and Hall 1953):

$$
\beta_{h k l}=\beta_{\mathrm{D}}+\beta_{\mathrm{S}}=\frac{k \lambda}{D \cos \theta}+4 \varepsilon_{\mathrm{str}} \tan \theta
$$

multiplying the above equation by $\cos \theta$, gives

$$
\beta_{h k l} \cos \theta=\frac{k \lambda}{D}+4 \varepsilon_{\mathrm{str}} \sin \theta .
$$

To make a Williamson-Hall analysis, the term $\beta_{h k l} \cos \theta$ was plotted vs $4 \sin \theta$ for all orientation peaks of $\mathrm{ZnO}$ nanorods with the wurtzite hexagonal phase from
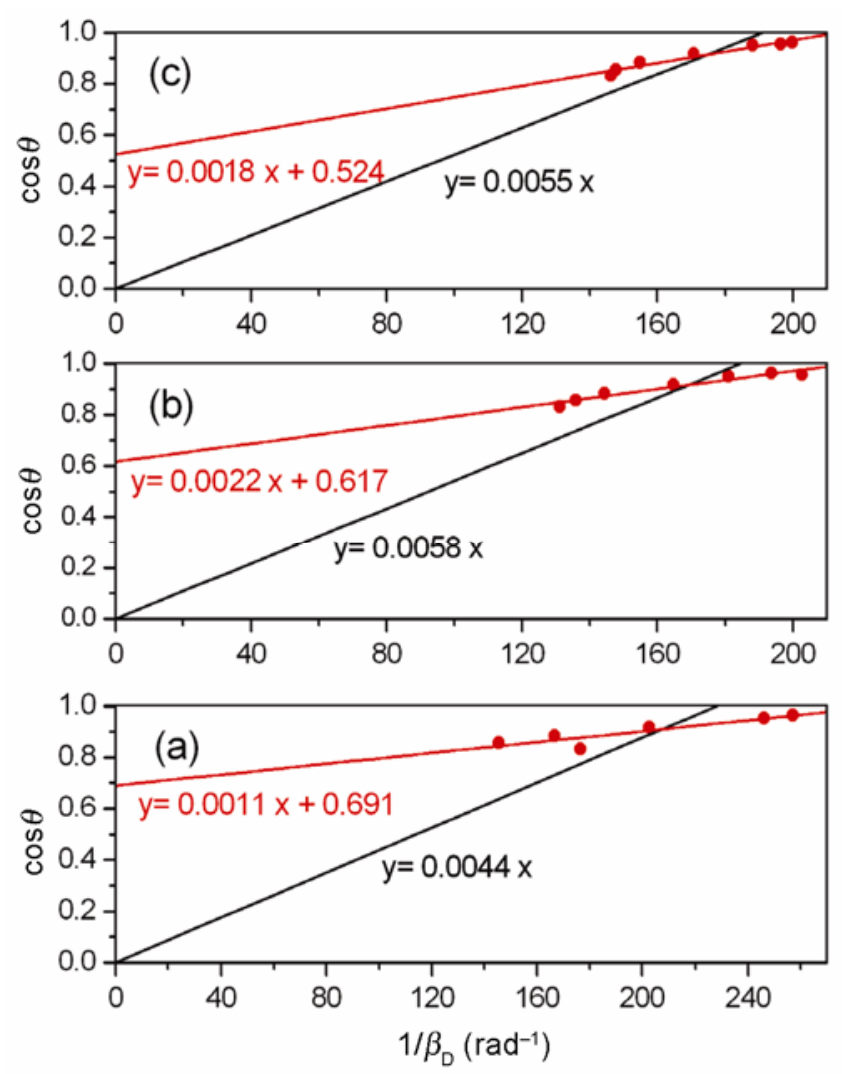

Figure 3. A comparison between two types of fit (normally linear fit and linear fit through zero) on Scherrer results of $\mathrm{ZnO}$ nanorods prepared by different hydrothermal growth times: (a) $5 \mathrm{~h}$, (b) $10 \mathrm{~h}$ and (c) $15 \mathrm{~h}$. Crystallite size $D$ is obtained from slope of fit. 
Table 2. Structure parameters of $\mathrm{ZnO}$ nanorods and average crystallite size estimated from averaging directly, slope of linear fit and slope of linear fit through zero.

\begin{tabular}{|c|c|c|c|c|c|c|c|c|}
\hline \multirow{2}{*}{$\begin{array}{l}\text { Hydrothermal } \\
\text { treatment time }(\mathrm{h})\end{array}$} & \multirow[b]{2}{*}{$h k l$} & \multirow[b]{2}{*}{$2 \theta\left(^{\circ}\right)$} & \multirow[b]{2}{*}{$d_{h k l}(\mathrm{~nm})$} & \multirow[b]{2}{*}{$\beta_{\mathrm{D}}\left(^{\circ}\right)$} & \multirow[b]{2}{*}{$D(\mathrm{~nm})$} & \multicolumn{3}{|c|}{ Average crystallite size $(\mathrm{nm})$ obtained by } \\
\hline & & & & & & Averaging & Linear fit & Linear fit through zero \\
\hline \multirow[t]{4}{*}{5} & & & & & & $35 \cdot 56$ & $131 \cdot 64$ & $33 \cdot 02$ \\
\hline & {$[100]$} & $31 \cdot 18$ & $0 \cdot 2813$ & $0 \cdot 223$ & $38 \cdot 64$ & & & \\
\hline & [002] & 33.88 & 0.2603 & 0.233 & 37.24 & & & \\
\hline & {$[101]$} & $35 \cdot 68$ & $0 \cdot 2476$ & $0 \cdot 283$ & $30 \cdot 81$ & & & \\
\hline \multirow[t]{4}{*}{10} & & & & & & $29 \cdot 1$ & $65 \cdot 82$ & $26 \cdot 71$ \\
\hline & {$[100]$} & $31 \cdot 3$ & $0 \cdot 2808$ & $0 \cdot 296$ & $29 \cdot 12$ & & & \\
\hline & [002] & 33.95 & $0 \cdot 2597$ & $0 \cdot 283$ & $30 \cdot 67$ & & & \\
\hline & {$[101]$} & $35 \cdot 75$ & $0 \cdot 2471$ & $0 \cdot 317$ & $27 \cdot 51$ & & & \\
\hline \multirow[t]{4}{*}{15} & & & & & & $29 \cdot 45$ & $80 \cdot 45$ & $27 \cdot 65$ \\
\hline & {$[100]$} & $31 \cdot 28$ & $0 \cdot 2809$ & $0 \cdot 287$ & $30 \cdot 03$ & & & \\
\hline & {$[002]$} & 33.93 & $0 \cdot 2599$ & $0 \cdot 292$ & $29 \cdot 72$ & & & \\
\hline & [101] & $35 \cdot 70$ & $0 \cdot 2472$ & 0.305 & 28.59 & & & \\
\hline
\end{tabular}
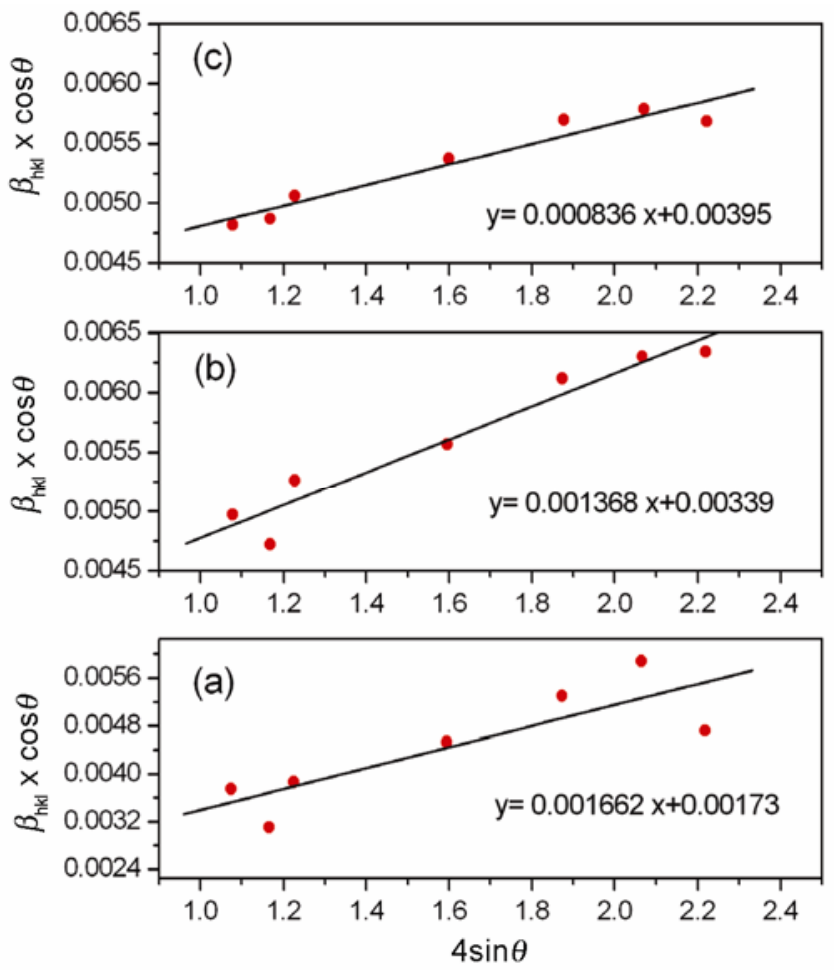

Figure 4. $\mathrm{W}-\mathrm{H}$ analysis of $\mathrm{ZnO}$ nanorods prepared by different hydrothermal growth times: (a) $5 \mathrm{~h}$, (b) $10 \mathrm{~h}$ and (c) $15 \mathrm{~h}$ assuming UDM. Crystallite size and strain are determined from slope and $y$-intercept of fit, respectively.

$2 \theta=20^{\circ}$ to $2 \theta=80^{\circ}$ (figure 4 ). By fitting a straight line to the data, the crystallite size and strain are determined from the slope and $y$-intercept of the fit, respectively. Equation (3) is written for the isotropic nature of crystal and represents UDM, where the strain in the material is assumed to be uniform in all crystallographic directions. The results obtained from UDM analysis for $\mathrm{ZnO}$ nanorods are shown in figure 4 and table 3.
In the uniform stress deformation model (USDM), there is a linear proportionality between stress and strain given by $\sigma=Y \varepsilon$ which is known as the Hook's law within the elastic limit.

In this relation, $\sigma$ is the stress of the crystal and $Y$ the Young's modulus. This equation is valid only for small deformations. By assuming a small strain in the lattice of $\mathrm{ZnO}$ nanorods, Hook's law is a reasonable approximation to estimate the lattice stress. By supposing the uniformity of small deformation stress in all crystallographic directions, replacing the value of $\varepsilon$ in the second term of (4) yields:

$$
\beta_{h k l} \cos \theta=\frac{k \lambda}{D}+4 \frac{\sigma}{Y_{h k l}} \sin \theta,
$$

$Y_{h k l}$ is the Young's modulus in the direction normal to the set of crystal lattice plane $h \mathrm{kl}$. In a hexagonal crystal, Young's modulus is given by (Zhang et al 2006):

$$
Y_{h k l}=\frac{\left[h^{2}+\frac{(h+2 k)^{2}}{3}+\left(\frac{a l}{c}\right)^{2}\right]^{2}}{\left[s_{11}\left(h^{2}+\frac{(h+2 k)^{2}}{3}\right)^{2}+s_{33}\left(\frac{a l}{c}\right)^{4}\right]},
$$

where $s_{11}, s_{33}, s_{44}$ and $s_{13}$ are the elastic compliances of $\mathrm{ZnO}$ with the standard values of $-2.206 \times 10^{-12}, 6.940 \times$ $10^{-12}, 23.57 \times 10^{-12}$ and $7 \cdot 858 \times 10^{-12}$, respectively (Nye 1985). Young's modulus was calculated as $\sim 130 \mathrm{MPa}$. As shown in figure 5 , plots were drawn with $(4 \sin \theta) / Y_{h k l}$ along the $x$-axis and $\beta_{h k l} \cos \theta$ along the $y$-axis for $\mathrm{ZnO}$ nanorods hydrothermally treated for different durations. The uniform deformation stress was calculated from the slope of the linear fit. 
Table 3. Geometric parameters including crystallite size and microstrain properties obtained from Scherrer relation, WilliamsonHall and Halder-Wagner analyses for $\mathrm{ZnO}$ nanorods prepared by hydrothermal heating for $5 \mathrm{~h}, 10 \mathrm{~h}$ and $15 \mathrm{~h}$.

\begin{tabular}{|c|c|c|c|c|c|c|c|c|c|c|c|c|}
\hline \multirow{3}{*}{$\begin{array}{l}\text { Hydrothermal } \\
\text { treatment } \\
\text { time (h) }\end{array}$} & \multirow{3}{*}{$\begin{array}{l}\text { Scherrer } \\
\text { method, } D \\
\quad(\mathrm{~nm})\end{array}$} & \multicolumn{9}{|c|}{ Williamson-Hall method } & \multirow{2}{*}{\multicolumn{2}{|c|}{$\begin{array}{l}\text { Halder-Wagner } \\
\text { method }\end{array}$}} \\
\hline & & \multicolumn{2}{|c|}{ UDM } & \multicolumn{3}{|c|}{ USDM } & \multicolumn{4}{|c|}{ UDEDM } & & \\
\hline & & $\begin{array}{c}D \\
(\mathrm{~nm})\end{array}$ & $\varepsilon \times 10^{-3}$ & $\begin{array}{c}D \\
(\mathrm{~nm})\end{array}$ & $\varepsilon \times 10^{-3}$ & $\begin{array}{c}\sigma \\
(\mathrm{MPa})\end{array}$ & $\begin{array}{c}D \\
(\mathrm{~nm})\end{array}$ & $\varepsilon \times 10^{-3}$ & $\begin{array}{c}\sigma \\
(\mathrm{MPa})\end{array}$ & $\begin{array}{c}u \\
\left(\mathrm{k} \mathrm{Jm}^{-3}\right)\end{array}$ & $\begin{array}{c}D \\
(\mathrm{~nm})\end{array}$ & $\varepsilon \times 10^{-3}$ \\
\hline 5 & $33 \cdot 02$ & $83 \cdot 49$ & $1 \cdot 66$ & $84 \cdot 31$ & $1 \cdot 68$ & $213 \cdot 36$ & $83 \cdot 46$ & $1 \cdot 66$ & $210 \cdot 82$ & 174.98 & $59 \cdot 33$ & $7 \cdot 13$ \\
\hline 10 & $26 \cdot 71$ & $42 \cdot 72$ & $1 \cdot 37$ & $42 \cdot 83$ & $1 \cdot 40$ & $177 \cdot 80$ & $42 \cdot 71$ & $1 \cdot 39$ & $176 \cdot 53$ & $122 \cdot 69$ & $35 \cdot 21$ & $6 \cdot 67$ \\
\hline 15 & $27 \cdot 65$ & $36 \cdot 66$ & $0 \cdot 84$ & $36 \cdot 47$ & $0 \cdot 84$ & $106 \cdot 68$ & $36 \cdot 40$ & $0 \cdot 83$ & $105 \cdot 41$ & $43 \cdot 74$ & $35 \cdot 34$ & $5 \cdot 51$ \\
\hline
\end{tabular}
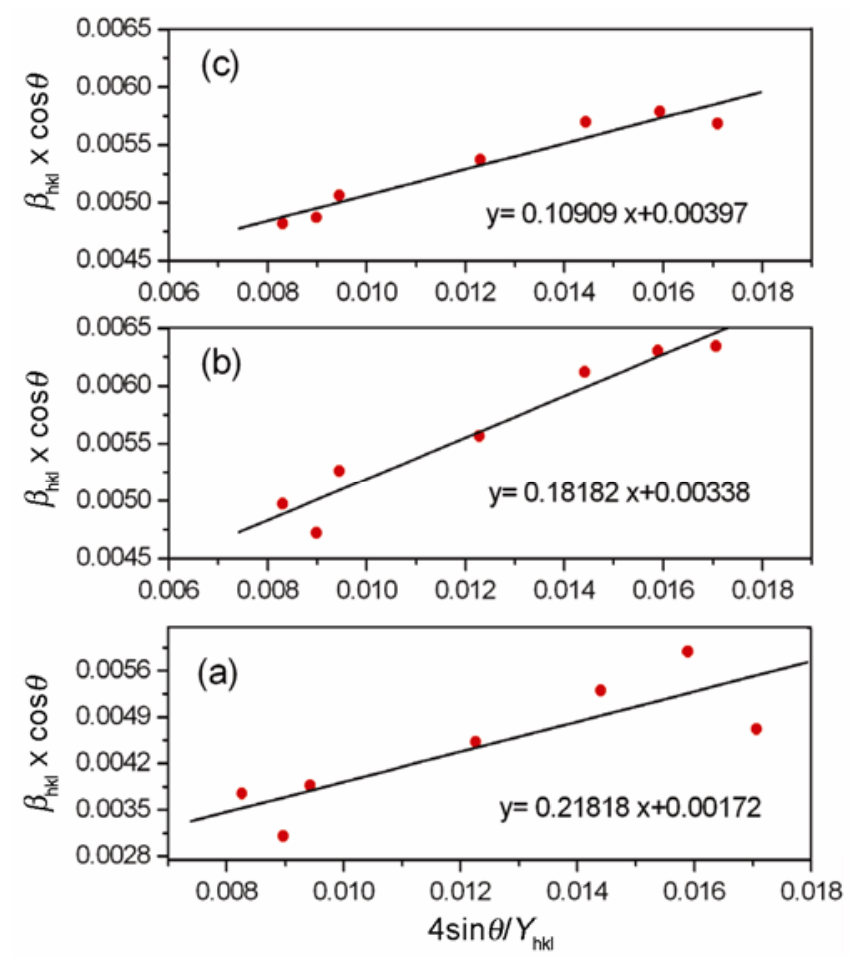

Figure 5. $\mathrm{W}-\mathrm{H}$ analysis of $\mathrm{ZnO}$ nanorods prepared by different hydrothermal growth times: (a) $5 \mathrm{~h}$, (b) $10 \mathrm{~h}$ and (c) $15 \mathrm{~h}$ assuming USDM. Uniform deformation stress is calculated from slope of linear fit.

In (4), the homogenous isotropic nature of the crystal is considered, whereas in many cases this assumption is not fulfilled. Moreover, the constants in stress-strain relation are no longer independent when the strain energy density $u$ is taken into account. According to the Hook's law, the relation between energy density $u$ and strain is given by $u=\left(\varepsilon^{2} Y_{h k l}\right) / 2$. Therefore, by replacing $u$ in (4), the relation is modified to the form:

$$
\beta_{h k l} \cos \theta=\frac{k \lambda}{D}+\left(4 \sin \theta\left(\frac{2}{Y_{h k l}}\right)^{1 / 2}\right) u^{1 / 2} .
$$

The uniform deformation energy density in UDEDM was obtained from the plot drawn between $\beta_{h k l} \cos \theta$ and $4 \sin \theta$ $\left(2 / Y_{h k l}\right)^{1 / 2}$ (figure 6). The value of $u$ was calculated from the slope and the crystallite size is estimated from the $y$ intercept of linear fit. The results obtained from the Scherrer method and W-H models (UDM, USDM and UDEDM) are summarized in table 3 . The values of strain is lower than the values obtained for $\mathrm{ZnO}$ nanorods that have been made by other techniques like chemical solution method (Sharma et al 2011).

Although both USDM and UDEDM consider the anisotropic nature of the crystallites, which is not similar to UDM approach, there is no considerable difference in strain values (table 3 ). Moreover, it can be clearly seen that considering the uniformity of deformation energy density in (7) did not affect the values for lattice strain, stress and crystallite size at all.

On the other hand, the value of crystallite size calculated by Scherrer method is less than that obtained by Williamson-Hall method. This difference is attributed to the strain value and confirms that the role of strain is important; therefore, it should be considered in the calculation of crystalline size. Thus, using the Scherrer method without considering strain may yield inaccurate results.

The lattice strain calculated by Williamson-Hall plot of XRD line profile showed a decrease with the increase in hydrothermal treatment time. Growth mechanism generally induces some strains on the crystal. When the growth time of rods increased, the domains structure broke and released the extra strain and in this way, a crystallite size reduction was obtained by increasing hydrothermal treatment time (table 3 ). Changing the preferred orientation of nanorods implied that nanorods experienced recrystallization and the corresponding strain releasing with increasing hydrothermal treatment time.

3.2c Halder-Wagner method: According to the previous works, Williamson-Hall plot showed the isotropic nature of line broadening and diffracting domains and existence of a microstrain contribution. However, if the Williamson-Hall plot does not indicate a marked anisotropy of size and strain broadening and the line profiles 
are described by the symmetric Voigt function, the sizestrain parameters can be well obtained by considering an average size-strain plot. Halder and Wagner (1966) have given an approximation to the integral breadth of a Voigt function as:

$$
\beta_{h k l}^{2}=\beta_{\mathrm{L}} \beta_{h k l}+\beta_{\mathrm{G}}^{2},
$$

where $\beta_{\mathrm{L}}$ and $\beta_{\mathrm{G}}$ are the Lorentzian and Gaussian components, respectively. In Halder-Wagner method, the crystallite size and strain profiles are described by the Lorentzian and Gaussian functions, respectively (Langford 1992). Consequently, we have

$$
\left(\frac{\beta_{h k l}^{*}}{d_{h k l}^{*}}\right)^{2}=\frac{1}{D}\left(\frac{\beta_{h k l}^{*}}{d_{h k l}^{* 2}}\right)+\left(\frac{\varepsilon}{2}\right)^{2},
$$

where $\beta_{h k l}^{*}=\beta_{h k l} \cos \theta l \lambda$ and $d_{h k l}^{*}=2 d_{h k l} \sin \theta l \lambda$. Similarly to Williamson-Hall method, the term $\left(\beta_{h k l}^{*} / d_{h k l}^{*}\right)^{2}$ was plotted versus $\left(\beta{ }_{k} k l / d_{k k l}^{*}\right)$ for all reflection peaks of $\mathrm{ZnO}$ nanorods. From linear fitted data to the plot, $y$-intercept gives the mean value of the strain and the slope gives the crystallite size. This plot has the advantage that data for reflections at low and mid-angles
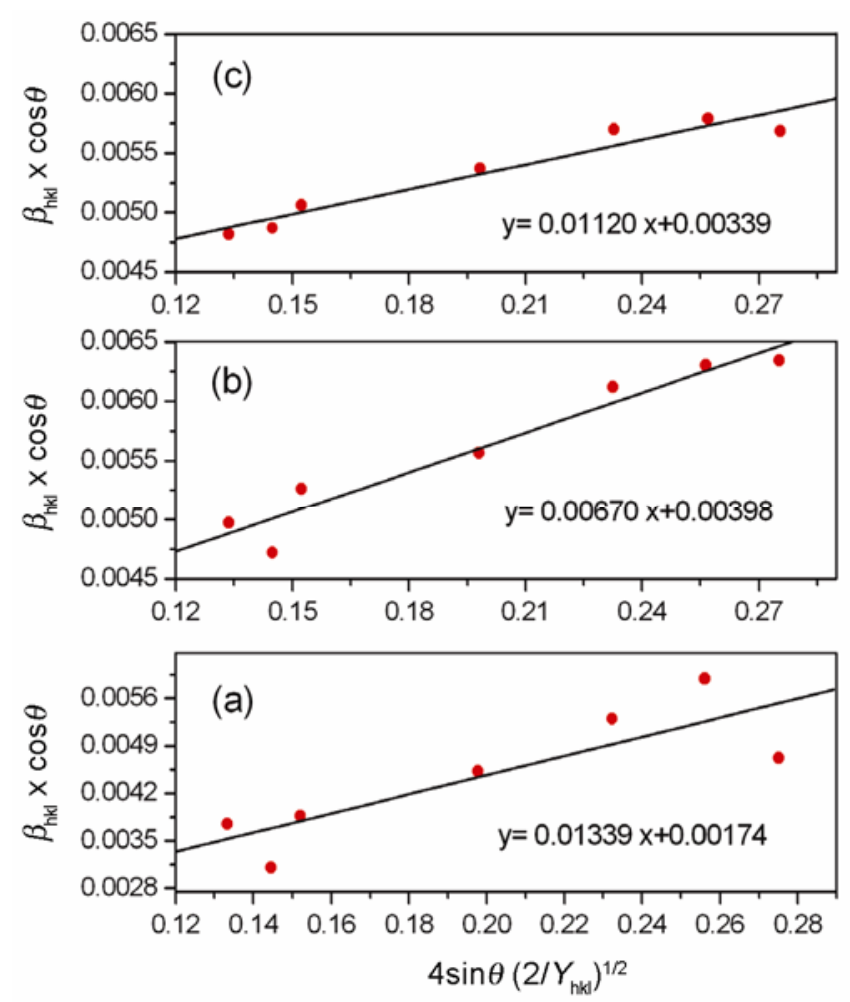

Figure 6. $\mathrm{W}-\mathrm{H}$ analysis of $\mathrm{ZnO}$ nanorods prepared by different hydrothermal growth times: (a) $5 \mathrm{~h}$, (b) $10 \mathrm{~h}$ and (c) $15 \mathrm{~h}$ assuming UDEDM. Value of uniform deformation energy density $u$ and crystallite size are calculated from slope and $y$-intercept of linear fit made to graph, respectively. are given more weight than those at higher angles where the precision is often lower. Halder-Wagner plots and crystallite size and strain obtained for $\mathrm{ZnO}$ nanorods are given in figure 7 and table 3. As shown by HalderWagner plots for the three samples, $\beta_{h k l}^{*} / d_{h k l}^{2}$ linearly increases with $\left(\beta_{h k l}^{*} / d_{h k l}\right)^{2}$ for all reflections with a positive slope and a non-zero $y$-intercept. This implies the existence of microstrains and spherical crystallite shape.

For estimating the crystallite size and strain, an appropriate method criterion is defined as the lesser scattering of the points from the fitting line. As shown in figures 47 , it is observed that the scattering of the points from the linear expression is lesser for the samples which are hydrothermally treated for 10 and $15 \mathrm{~h}$. The data points for the sample which are hydrothermally treated for $5 \mathrm{~h}$ has a very large scatter indicating anisotropy. Therfore, the accuracy of $\mathrm{W}-\mathrm{H}$ and Halder-Wagner plots for estimating size and strain contribution of diffraction profiles was reduced correspondingly.

As can be seen in table 3, the strain estimated by Halder-Wagner method was several times higher than that obtained by $\mathrm{W}-\mathrm{H}$ method. This considerable difference can be related to more contributions of lower angles in Halder-Wagner method. It seems that the lattice dislocations has significant effect on broadening of the reflection peaks situated at lower angles in comparison with higher angles.
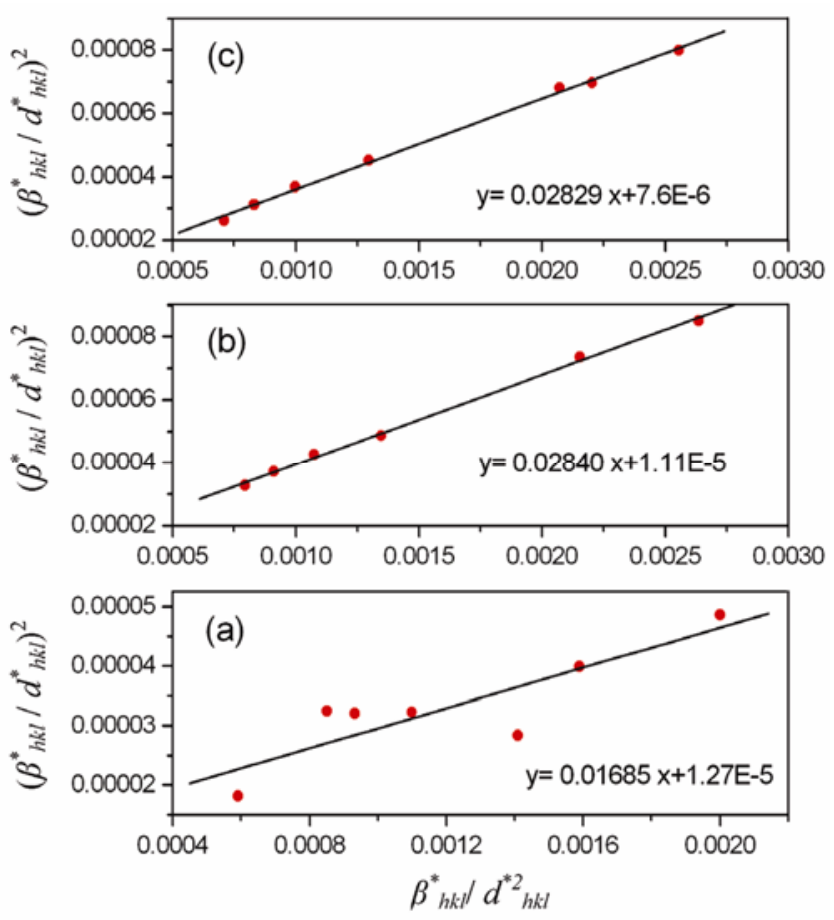

Figure 7. Halder-Wagner analysis of $\mathrm{ZnO}$ nanorods prepared by different hydrothermal growth times: (a) $5 \mathrm{~h}$, (b) $10 \mathrm{~h}$ and (c) 15 h. $y$-intercept gives mean value of strain and slope gives crystallite size. 

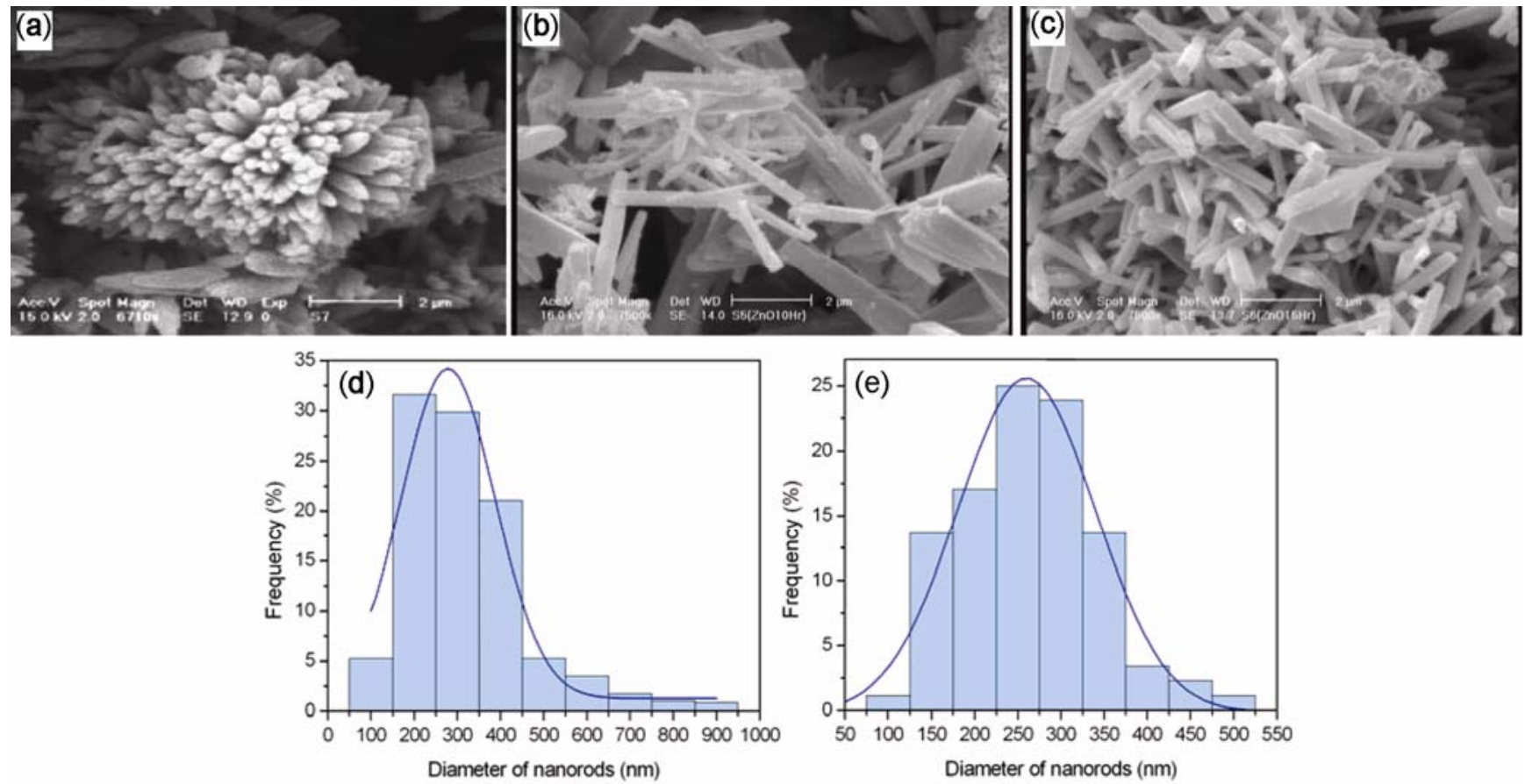

Figure 8. SEM images of $\mathrm{ZnO}$ nanorods fabricated by different hydrothermal treatment times: (a) $5 \mathrm{~h}$, (b) $10 \mathrm{~h}$ and (c) $15 \mathrm{~h}$. Bar graphs show diameter size distribution of samples consisting of single nanorods prepared after (d) $10 \mathrm{~h}$ and (e) $15 \mathrm{~h}$.

In the case of crystallite size, apart from noticeable differences in the values obtained by three methods, the trend of changes observed in Halder-Wagner method was similar to that estimated by the Scherrer's equation rather than $\mathrm{W}-\mathrm{H}$ method.

\subsection{Morphology analysis}

SEM micrographs of the hydrothermally heated samples for 5,10 and $15 \mathrm{~h}$ are shown in figure 8 . As shown in figure 8(a), the microflower type structure was formed in the $5 \mathrm{~h}$ hydrothermally heated sample by accumulation of hundreds of $\mathrm{ZnO}$ nanorods. The typical lengths of these nanorods were in the range of $1-2 \mu \mathrm{m}$ and became thinner from root to the top end to form a sharp edge at the tip. All the $\mathrm{ZnO}$ nanorods were attached together through their thicker bases to form the microflower with the full array size in the range of $2-4 \mu \mathrm{m}$. Shaping the microflower can be attributed to the high rate formation of $\mathrm{Zn}(\mathrm{OH})_{2}$ particles as nucleating sites in the solution. When the hydrothermal treatment time reached $10 \mathrm{~h}$, the microflower collapsed and recrystallized to nanorods (figure $8(\mathrm{~b})$ ). In this case, the nucleation rate decreased and nucleation preferably occurred on $c$-face of the crystallites, since this orientation reached the critical size for island nucleation. This process was repeated to form rodlike nanostructures. As can be seen in figure 8(b) and the histogram of nanorods diameter size distribution in figure $8(d)$, synthesized nanorods are very much different in length and diameter. By prolonging the hydrothermal heating process to $15 \mathrm{~h}$, the structures were broken down and re-growth to [101]-oriented hexagonal nanorods with the smooth surface and no nanoparticles were observed to be attached to them (figure $8(\mathrm{c})$ ). The length of the grown nanorods were in the range of $1.5-2.5 \mu \mathrm{m}$ whereas the diameter of more than $50 \%$ of $\mathrm{ZnO}$ nanorods varied from 225 to $325 \mathrm{~nm}$ (figure 8(e)). From the above observations, it can be concluded that the morphological characteristics of $\mathrm{ZnO}$ nanostructures are controlled by hydrothermal treatment time.

\section{Conclusions}

In summary, $\mathrm{ZnO}$ nanorods and microflower were fabricated by a simple, low temperature and efficient hydrothermal method without using any template, substrate, catalyst, nucleation controlling agent and autoclave. As compared to the conventional hydrothermal method, the present synthesis process has the advantage of simplicity and low cost, low growth temperature $\left(90^{\circ} \mathrm{C}\right)$, controlling morphology and crystallization orientation, high morphological yield (nearly 100\%) and low crystal strain. This work highlights the importance of hydrothermal treatment time $(5,10$ and $15 \mathrm{~h})$ on the morphology and preferable crystal orientation of $\mathrm{ZnO}$ nanorods. $\mathrm{ZnO}$ nanorods exhibited wurtzite structure with preferred crystallographic orientation of [101]. By $10 \mathrm{~h}$ hydrothermal heating, the single nanorods were obtained but the cross- 
section diameter size distribution was not uniform at all. The preferential orientation of [002] facets has been observed in the case of nanorods. After $5 \mathrm{~h}$ hydrothermal heating, $\mathrm{ZnO}$ microflower consisting of the nanorods with length in the range of $1-2 \mu \mathrm{m}$ was formed. Nanorods became thinner from root to the top end to form a sharp edge at the tip. Finally, after $15 \mathrm{~h}$ hydrothermal treatment time, single $\mathrm{ZnO}$ nanorods with diameter in the range of $100-500 \mathrm{~nm}$ were preferably grown along the [101] direction.

XRD analysis including crystallite size, microstrain and Young's modulus measurements were performed by Scherrer, Williamson-Hall and Halder-Wagner methods. It was concluded from XRD observations that the contribution of microstrain in XRD broadening cannot be ignored. It was also observed that with increasing hydrothermal treatment time, defects in lattice considerably decreased.

\section{References}

Aneesh P M, Vanaja K A and Jayaraj M K 2007 Proc. SPIE $663966390 \mathrm{~J}$

Baruah S and Dutta J 2009 J. Sol-Gel Sci. Technol. 50456

Brahma S, Rao K J and Shivashankar S 2010 Bull. Mater. Sci. 3389

Chander R and Raychaudhuri A K 2006 J. Mater. Sci. 413623

Chen D R, Jiao X L and Cheng G 1999 Solid State Commun. 113363

Dhara S and Giri P K 2011 Appl. Nanosci. 1165

Ebrahimizadeh Abrishami M, Kompany and Hosseini S M 2012 J. Electroceram. 29125

Ebrahimizadeh Abrishami M and Hosseini S M 2013 J. Mater Sci.: Mater. Electron. 2464

Ebrahimizadeh Abrishami M and Kompany A 2012 Adv. Mater. Res. 462201

Ghosh M, Bhattacharyya R and Raychaudhuri 2008 Bull. Mater. Sci. 31283

Halder C and Wagner C N J 1966 Acta Cryst. 20312

He Y 2005 J. Mater. Lett. 59114

Hingorani S, Pillai V, Kumar P, Muntai M S and Shah D O 1993 Mater. Res. Bull. 281303

Huang M et al 2001 Sciences 2921897

Khorsand Zak A, Majid W H Abd, Ebrahimizadeh Abrishami M and Yousefi R 2011 Solid State Sci. 13251
Khorsand Zak A, Majid W H Abd, Ebrahimizadeh Abrishami M, Yousefi R and Parvizi R 2012 Solid State Sci. 14488

Kim N-H, Jung S-H, Park J H, Lee K-H and Cho K 2011 IEEE Trans. Nanotech. 101347

Kim Y J, Shang H and Cao G 2006 J. Sol-Gel Sci. Technol. 38 79

Ko Y H, Kim M S and Yu J S 2012 Appl. Surf. Sci. 25999

Langford J I 1992 NIST Spec. Publ. 846110

Li Y, Meng G W and Zhang L D 2000 Appl. Phys. Lett. 76 2011

Lipowsky P, Hirscher M, Hoffmann R C and Bill J 2007 Nanotechnology 18165603

Liu Y, Liu A, Liu W, Hu Z and Sang Y 2010 Appl. Surf. Sci. 2571263

Miao L, Zhang H, Zhu Y, Yang Y, Li Q and Li J 2012 J. Mater. Sci.: Mater Electron. doi: 10.1007/s10854-012-0679-8

Navaladian S, Viswanathan B, Varadarajan T K and Viswanath R P 2009 Nanoscale Res. Lett. 4471

Nye J F 1985 Physical properties of crystals: their representation by tensors and matrices (New York: Oxford)

Panda S K and Jacob C 2009 Bull. Mater. Sci. 32493

Park W I, Kim D H, Jung S W and Yi G C 2002 Appl. Phys. Lett. 804232

Rogers K D and Daniels P 2002 Biomaterials 232577

Sakohara S, Ishida and Anderson M A 1998 J. Phys. Chem. B102 10169

Sharma B K, Khare N, Kumar Mahesh and Kumar P 2011 Chem. Phys. Lett. 51562

Sharma P et al 2003 Nature Materials 2673

Sima M, Vasile E and Sima M 2012 Thin Solid Films 5204632

Sōmiya S and Roy R 2000 Bull. Mater. Sci. 23453

Stokes A R and Wilson A J C 1944 Proc. Phys. Soc. London 56 174

Umar A, Karunagaran B, Suh E-K and Hahn Y B 2006 Nanotechnology 174072

Williamson G K and Hall W H 1953 Acta Metall. 122

Yao B D, Chan Y F and Wang N 2002 Appl. Phys. Lett. 81 757

Yuan Z, Yu J, Wang N and Jiang Y 2011 J. Mater Sci.: Mater. Electron. 221730

Yuan Z, Yu J and Jiang Y 2011 Energy Procedia 12502

Zhang J, Zhang Y, Xu K W and Ji V 2006 Solid State Commun. 13987

Zhao X, Zhang S C, Li C, Zheng B and Gu H 1997 J. Mater. Synth. Process 5227

Zhu F, Zhang Y, Yan Y, Song W and Xia L 2008 Bull. Mater. Sci. 31121 